\title{
Angola's 2013 dengue outbreak: clinical, laboratory and molecular analyses of cases from four Portuguese institutions
}

\author{
Ricardo Parreira ${ }^{1}$, Cláudia Conceição ${ }^{2}$, Sónia Centeno-Lima ${ }^{2,3}$, Nuno Marques ${ }^{4}$, José Saraiva da \\ Cunha $^{4}$, Cândida Abreu ${ }^{5}$, Laura Sá ${ }^{5}$, António Sarmento ${ }^{5}$, Jorge Atouguia ${ }^{2,3}$, Virgínia Moneti ${ }^{6}$, Telma \\ Azevedo $^{6}$, Jaime Nina ${ }^{2,6}$, Kamal Mansinho ${ }^{2,6}$, Abílio Antunes ${ }^{7}$, Rosa Teodósio ${ }^{2}$, Teresa Nazareth ${ }^{2,8}$, \\ Jorge Seixas ${ }^{2}$ \\ ${ }^{1}$ Unidade de Microbiologia Médica (Grupo de Virologia) and Unidade de Parasitologia e Microbiologia Médicas \\ (UPMM), Instituto de Higiene e Medicina Tropical (IHMT), Universidade Nova de Lisboa (UNL), Lisboa, Portugal \\ ${ }^{2}$ Unidade de Clínica Tropical, IHMT/UNL, Lisboa, Portugal \\ ${ }^{3}$ Centro de Malária e Outras Doenças Tropicais (CMDT), IHMT/UNL, Lisboa, Portugal \\ ${ }^{4}$ Centro Hospitalar e Universitário de Coimbra, Unidade de Doenças Infecciosas, Coimbra, Portugal \\ ${ }^{5}$ Centro Hospitalar de São João, Porto, Portugal \\ ${ }^{6}$ Centro Hospitalar de Lisboa Ocidental/Hospital de Egas Moniz, Serviço de Doenças Infecciosas, Portugal \\ ${ }^{7}$ Associação para o Desenvolvimento da Medicina Tropical/IHMT, Lisboa, Portugal \\ ${ }^{8}$ Unidade de Parasitologia Médica, IHMT/UNL, Lisboa, Portugal
}

\begin{abstract}
Introduction: Dengue virus (DENV) is the arbovirus with the widest impact on human health. In Africa in general, and in Angola in particular, the epidemiology and public health impact of DENV is far from clear. However, rapid population growth, unplanned urbanization, increased international travel, and the presence of virus major vector (Aedes aegypti) in the country suggest that DENV transmission may occur.

Methodology: In parallel to the occurrence of a dengue outbreak affecting the capital of Angola, between March and July 2013 four Portuguese institutions diagnosed dengue infection in 146 individuals returning to Portugal. Clinical presentation, laboratory findings, and molecular analyses of partial viral genomic segments were performed.

Results: The mean age of the individuals included in this study was 42 years old, the majority being men of Portuguese nationality, reporting various lengths of stay in Angola. Fever was the most reported clinical sign, being frequently associated (61.0\%) with myalgia and headache. Hematological values, including hematocrit, white-blood cell and platelets counts, correlated with the absence of severe or complicated cases, or coagulation disorders. No deaths were observed. Viral NS1 was detected in $56.2 \%$ of the samples, and all NS1 negative cases had antidengue IgM antibodies. RT-PCR indicated the presence of DENV1, which was confirmed by phylogenetic analysis of 25 partial NS5 viral sequences.

Conclusion: The DENV cases analyzed conformed to classical and uncomplicated dengue, caused by the suggested exclusive circulation of a genetically homogeneous DENV1 of genotype III, apparently with a single origin.
\end{abstract}

Key words: Dengue virus; imported viral diseases; laboratory surveillance; outbreaks.

J Infect Dev Ctries 2014; 8(9):1210-1215. doi:10.3855/jidc.4910

(Received 24 February 2014 - Accepted 27 June 2014)

Copyright (C) 2014 Parreira et al. This is an open-access article distributed under the Creative Commons Attribution License, which permits unrestricted use, distribution, and reproduction in any medium, provided the original work is properly cited.

In recent decades, dengue virus (DENV) has emerged as a worldwide public health problem. Although its real impact on human health is difficult to assess, estimates refer that 50-400 million infections may occur worldwide annually $[1,2]$. The majority of these are asymptomatic, and symptomatic cases present as an acute and abrupt febrile syndrome (classical dengue or dengue fever). However, some may evolve to potential life threatening clinical presentations (dengue hemorrhagic fever/dengue shock syndrome) with a case-fatality rate of $0.1 \%-5 \%$ [3].

The geographical distribution of the DENVinfections is not homogeneous, the majority occurring in the Asia-Pacific and Americas-Caribbean regions [1]. In Africa, the epidemiology and public health impact of dengue is far from clear, but the wide geographical distribution of the virus's primary invertebrate vectors (Aedes aegypti and Aedes albopictus), rapid population growth, unplanned 
urbanization, and increased international travel, make the transmission of DENV very likely [4]. However, since malaria is endemic in most of the African continent, the great majority of febrile illnesses are diagnosed and treated as such, often without proper medical examination or laboratory confirmation [5]. In fact, serological surveys conducted in Burkina Faso [6] have suggested that dengue may be more prevalent in Africa than generally recognized.

In the context of an outbreak of dengue, first reported by the Angolan health authorities on the $1^{\text {st }}$ of April 2013 [7], a total of 146 dengue cases were identified in four Portuguese medical institutions between the $1^{\text {st }}$ of March and the $12^{\text {th }}$ of July, with a peak number of dengue cases in May $(n=70)$. These institutions included the Institute of Hygiene and Tropical Medicine (IHMT) and the Egas Moniz Hospital (HEM) in Lisbon, the University Hospital S. João (HSJ) in Oporto and the University Hospital (CHUC) in Coimbra. The mean age of the 146 individuals included in this study was 42 years old (ranging from 21 to 69 years), with $78.8 \%(\mathrm{n}=115)$ men (mean $=42.5$ years, ranging from 22 to 69 ) and $21.2 \%(\mathrm{n}=31)$ women (mean $=40.2$ years, ranging from 21 to 66$)$. The majority $(81.5 \%, \mathrm{n}=119)$ were of Portuguese nationality, the second most represented nationality was Angolan $(7.5 \% \mathrm{n}=11)$, while 5 individuals reported double nationality (3.4\%). Finally Spanish, Moldavan, and Romanian origin was reported once (each). Length of stay in Angola lasting up to 1 month was reported by 14 individuals, while 55 and 73 indicated having stayed in Angola $\leq 6$ months, and $>7$ months, respectively.

Dengue case definition included a suggestive clinical presentation (high fever, accompanied by two additional symptoms including severe headache, retroorbital pain, muscle and joint pains, nausea, vomiting, swollen glands or rash, as defined by WHO [1], along with a positive rapid diagnostic test (RDT). RDT results obtained, describing the detection of NS1 antigen, anti-DENV IgM and IgG antibodies, are collectively described in Figure 1. Cases with only anti-DENV IgG antibodies were excluded from the analysis. Fever was the most frequently reported clinical sign $(\mathrm{n}=133 / 91.0 \%)$, lasting, in the majority

Figure 1. Detection of NS1, IgM and IgG using RDT. The tests used were Panbio Dengue Early Rapid (NS1) and Dengue Duo (IgG/M) cassette, SD Bioline Dengue Duo (NS1 and $\mathrm{IgG} / \mathrm{M}$ ) and SD Bioline Dengue (IgG/M). NP indicates that RDT for NS1 detection was not performed as only the SD Bioline Dengue $(\mathrm{IgG} / \mathrm{M})$ was used for a period of time.

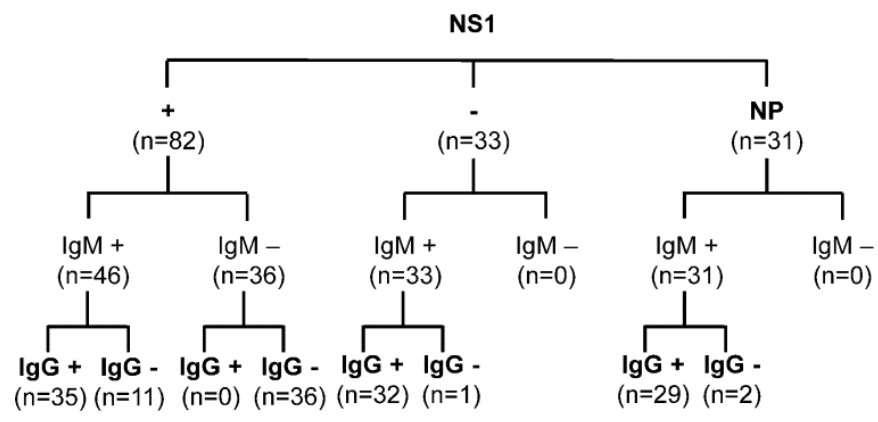

Table 1. Symptoms and signs associated with DENV infection

\begin{tabular}{cc}
\hline Symptoms and signs & $\mathbf{n} / \mathbf{0}$ \\
\hline fever & $133 / 91.0$ \\
myalgia & $113 / 77.3$ \\
headache & $99 / 67.8$ \\
asthenia & $74 / 50.7$ \\
arthalgia & $51 / 34.9$ \\
retro orbital pain & $40 / 27.4$ \\
diarrhea & $27 / 18.5$ \\
cutaneous rash & $19 / 13.0$ \\
\hline
\end{tabular}

Table 2. Hematological parameters associated with DENV infection.

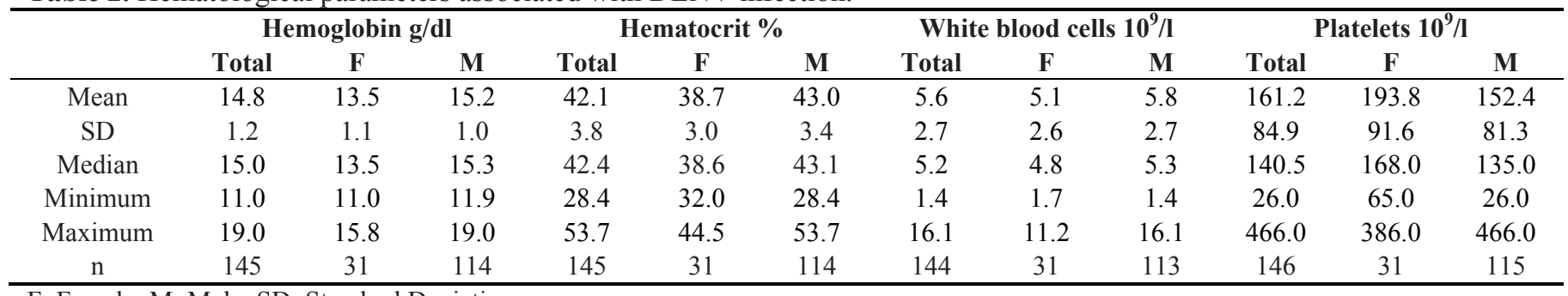

F: Female; M: Male; SD: Standard Deviation. 
(70.2\%) of the cases (59 out of 84 for which the feverish period was recorded) between 4 and 7 days. The frequency of registered symptoms and signs is indicated in Table 1. The characteristic combination of fever, myalgia and headache was present in $61.0 \%$. No clinical evidence of coagulation disorder was observed. Malaria was ruled out in febrile patients either by direct examination (at the CHUC) or by direct examination and Plasmodium sp. RDT (at IHMT, HEM, HSJ). In 8 cases, a malaria test was

Figure 2. Phylogenetic analysis of DENV NS5 sequences. The tree was constructed using genetic distances corrected with the TN93 model and the neighbor-joining clustering algorithm. Reference sequences are indicated by geographic origin and viral strain name (or date, in the case of the Angolan $_{2013}$ cluster). Bootstrap values over $87 \%$ are indicated. The size bars indicates 0.05 nucleotide substitutions per site.

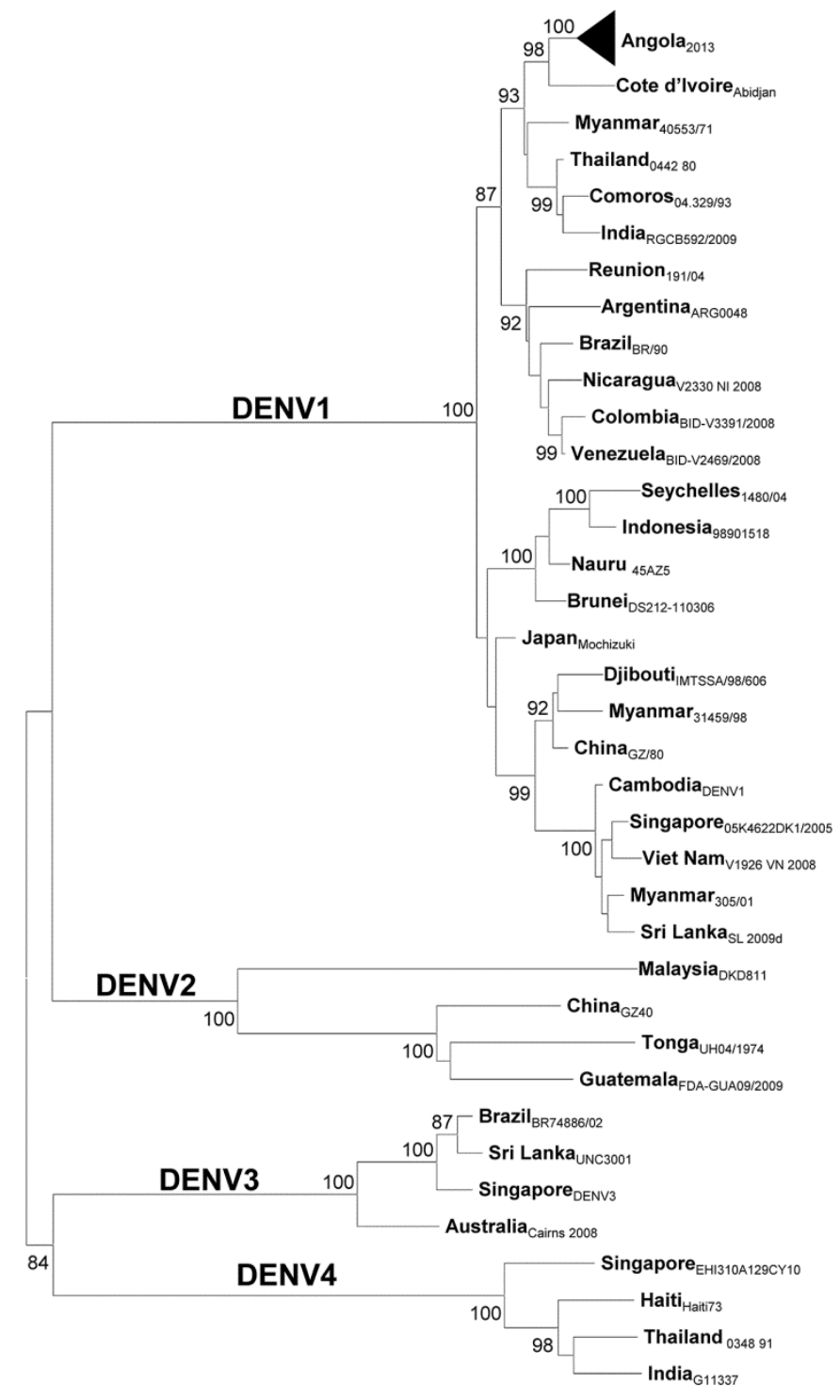

0.05 performed in another institution. In 5 additional patients, malaria was excluded based only on clinical grounds (e.g. incubation period or previous recent malaria treatment in Angola). Hematological data is shown in Table 2. The values indicating the average amount of hemoglobin and hematocrit together with white-blood cell and platelets counts correlated with the absence of severe or complicated cases, as defined by WHO [1]. No deaths were observed.

Viral NS1 was detected in $56.2 \%$ of the samples (n $=82)$. All NS1 negative cases $(n=33)$ had antidengue IgM antibodies. In the 31 cases where NS1 detection was not carried out, 29 showed $\operatorname{IgM}$ and IgG anti-dengue antibodies, whereas 2 cases had only IgM (Figure 1).

Detection of viral RNA was carried out by nested RT-PCR using primers D1 and D2 in the first-round and D1 with a mixture of subtype-specific reverse primers (TS1-4) in a multiplex second-round, as previously reported [8]. We analyzed a total of 25 samples (out of 29), the majority $(\mathrm{n}=24)$ also evidenced NS1 by RDT. As previously reported by IHMT [9], and in accordance to previously published studies [10] the amplicons size was compatible with only the presence of DENV1. Molecular confirmation was obtained by phylogenetic analysis of either C-prM (accession numbers HG515502-09) of NS5 (accession numbers HG515510-27) sequences, amplified using primers 1NS5F/1NS5R (first-round) and 2NS5F/2NS5R (second-round) as previously described [11]. The preliminary analysis of the obtained sequences (Figure 2) clearly indicates a segregation of the Angolan DENV sequences (collectively grouped as Angola $_{2013}$ ) in a consistent monophyletic cluster (bootstrap value of 100) within the DENV1 radiation (supporting the preliminary viral subtype assignment based on RT-PCR data), in association to a viral sequence of African origin (Cote d'Ivoire Abijan $_{\text {). }}$ Subsequently, a more robust Bayesian phylogenetic analysis (Figure 3) again confirmed, with maximum posterior probability, the monophyletic origin of the Angolan and Cote d'Ivoire sequences. Furthermore, the cluster formed by the Angolan sequences was characterized by low genetic variability, suggesting the circulation of viral strains sharing a common origin. As expected, the analysis of the putative products encoded by these sequences revealed overall sequence conservation, with only one nonconservative difference (indicated by the / symbol in Figure 4) between the Angolan $_{2013}$ Dengue NS5 sequence cluster and that of its most closely related

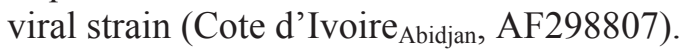


Figure 3. Bayesian phylogenetic tree analysis $\left(20 \times 10^{6}\right.$ generations using GTR $+\Gamma+\mathrm{I}$ model) of DENV1 NS5 sequences (accession numbers HG515510-HG515527) amplified from DENV-infected individuals returning from Angola during the 2013 spring outbreak. Reference strains, downloaded from the public databases, are indicated by geographic origin and strain name (when available). The branches referring to DENV1 genotypes 1-3 are indicated. Numbers at specific branches refer to high posterior probability values ( $>0.8)$. The size bars indicates 0.2 nucleotide substitutions per site.

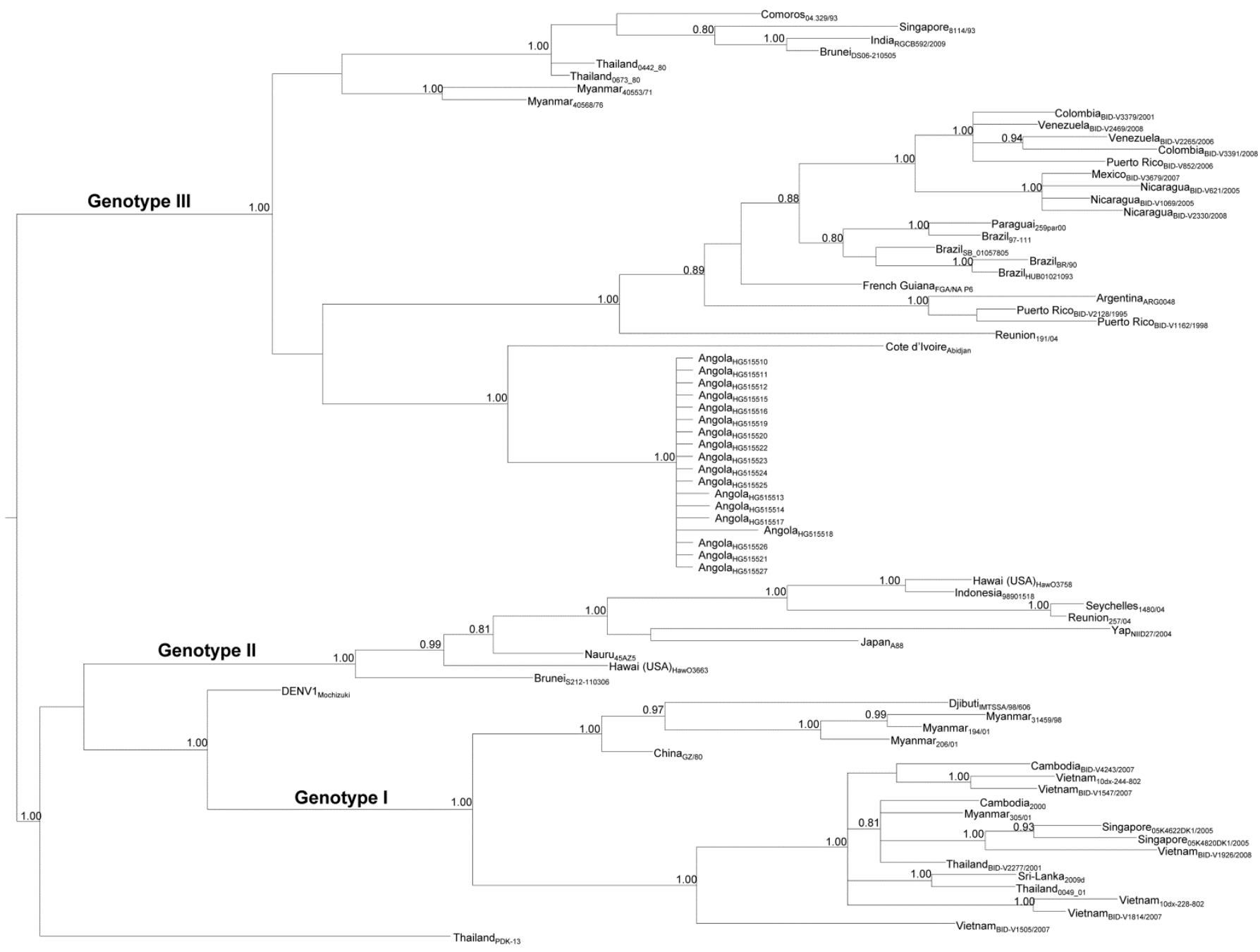

0.2

Figure 4. Multiple sequence alignment of the putative partial NS5 amino acid sequences obtained in the course of this work (indicated as Angola $_{2013}$ ) and that of DENV1 strain Cote d'Ivoire $_{\text {Abidjan }}$ (AF298807). Asterisks indicate identical amino acid residues. Conservative and non-conservative substitutions are indicated by: and /, respectively. Dots indicate sequence conservation. The numbers at the right-side of the figure indicate the number of each sequence type.
Angola $a_{2013}$ Angola $a_{2013}$ AF298807

Angola 2013 Angola $a_{2013}$ Angola 2013

Angola 2013

AF298807

Angola 2013

Angol $a_{2013}$

AF 298807

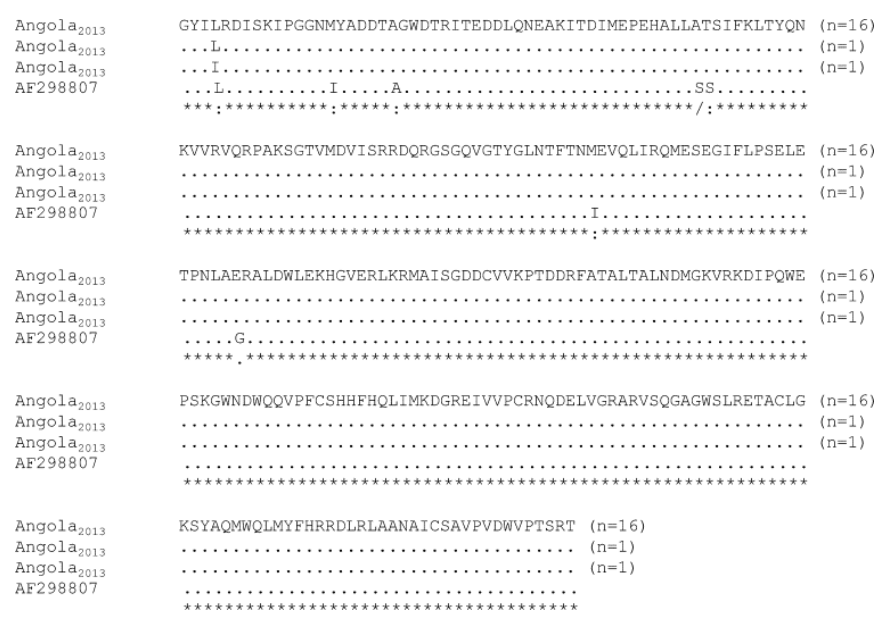


Aedes aegypti was identified in Angola in 1903, 1956 and 1973 [12-14]. Both serological reports from the 1960s [15], and the detection of DENV1 and DENV2 in travelers in the 1980s and in 1999/2002 $[16,17]$ suggest long lasting endemic DENV activity in the country. The origin of the DENV that was responsible for the outbreak in Luanda has been difficult to assess, and even the genetic analysis of the whole viral sequence [10] has not deemed possible a conclusive identification of its source, most probably due to limited sampling of viral strains of African origin. In turn, this consequently compromises subsequent genetic analyses.

The real number of dengue cases in Africa is surely under-reported due to i) low awareness of the medical community/health-care providers, ii) lack of laboratory testing, and iii) poor/nonexistent surveillance programs. In this context, travelers returning to their countries may serve as possible sentinels to local epidemics, especially in areas of the globe where surveillance of infectious agents is compromised. This issue is all the more relevant if we take into account the fact that a recent study indicated that approximately $16 \%$ of the total worldwide DENV infections occur in sub-saharan Africa [4]. Relevant to our analysis is the fact that DENV has been repeatedly found in travelers/expatriates returning from countries that border Angola such as the Democratic Republic of Congo and Namibia, although approximately half of the African countries where travelers had been infected with dengue do not report any local viral activity [4].

Long-distance travelers may allow the indirect reporting of transmission of infectious agents in areas where local detection is technically and/or financially unfeasible. Additionally, they may also serve as seeders of new outbreaks in case a viremic status is maintained upon arrival to their final destinations. Accordingly, the virus was detected in Germany, Canada, France, South-Africa and Israel [18]. In the context of Angola's 2013 outbreak, we were able to readily detect viral genomes by RT-PCR from Dengue-NS1reactive sera. This raises concerns regarding the potential endemic circulation of DENV in the territory, especially when a very recent report disclosed the presence of DENV in Luanda [19], as well as for establishment of epidemic foci in regions with active populations of Ae. aegypti and/or $A e$. Albopictus (e.g. Madeira Island and several countries in the Mediterranean basin) [20], opening the possibility for local virus introduction and dissemination from distant epidemic foci.

\section{References}

1. WHO (2008) Dengue and dengue haemorrhagic fever. Geneva, Switzerland. World Health Organization. Available at http://www.who.int/mediacentre/factsheets/fs117/en/. Accessed on 10/August/2014.

2. Bhatt S, Gething PW, Brady OJ, Messina JP, Farlow AW, Moyes CL, Drake JM, Brownstein JS, Hoen AG, Sankoh O, Myers MF, George DB, Jaenisch T, Wint GR, Simmons CP, Scott TW, Farrar JJ, Hay SI (2013) The global distribution and burden of dengue.Nature 496: 504-507.

3. WHO (2009) Dengue: guidelines for diagnosis, treatment, prevention and control. Geneva, Switzerland. World Health Organization. Available from http://whqlibdoc.who.int/publications/2009/9789241547871_ eng.pdf. Accessed on 10/August/2014.

4. Amarasinghe A, Kuritsk JN, Letson GW, Margolis HS (2011) Dengue virus infection in Africa. Emerg Infect Dis 17: 13491345.

5. Amexo M, Tolhurst R, Branish G, Bates I (2004) Malaria misdiagnosis: effects on the poor and vulnerable. Lancet 364 : 1896-1898.

6. Collenberg E, Ouedraogo T, Ganamé J, Fickenscher H, Kynast-Wolf G, Becher H, Kouyaté B, Kräusslich HG, Sangaré L, Tebit DM (2006) Seroprevalence of six different viruses among pregnant women and blood donors in rural and urban Burkina Faso: A comparative analysis. J Med Virol 78: 683-692.

7. Casos de dengue registados no hospital geral de Luanda. [Dengue cases recor5ded in the general hospital of Luanda]. ANGONOTÍCIAS. Article in Portuguese. Available from: http://www.angonotícias.com/Artigos/item/38122/casos-dedengue-registados-no-hospital-geral-de-luanda. Accessed on 7/May/2013.

8. Lanciotti RS, Calisher CH, Gubler DJ, Chang GJ, Vorndam AV (1992) Rapid detection and typing of dengue viruses from clinical samples by using reverse transcriptase-polymerase chain reaction. J Clin Microbiol 30: 545-551.

9. ProMED-mail. Dengue/DHF Update (31): Asia, Africa, Pacific. Archive Number: 20130420.1660193. 20 Apr 2013. Available from: http://www.promedmail.org.

10. Sessions OM, Khan K, Hou Y, Meltzer E, Quam M, Schwartz E, Gubler DJ, Wilder-Smith A (2013) Exploring the origin and potential for spread of the 2013 dengue outbreak in Luanda, Angola. Glob Health Action 6: 21822.

11. Vázquez A, Sánchez-Seco MP, Palacios G, Molero F, Reyes N, Ruiz S, Aranda C, Marqués E, Escosa R, Moreno J, Figuerola J, Tenorio A (2012) Novel flaviviruses detected in different species of mosquitoes in Spain. Vector Borne Zoonotic Dis 12: 223-229.

12. Roque B (1903) Contribution for thestudy of malaria and mosquitos from Angola [Article in Portuguese] A Medicina Contemporânea, Lisboa 22: 127-130.

13. Grândara AF (1956) Contribution for the study of «Culícidea» (Diptera) from Angola: Mission for the prospection of endemics. Anais do Instituto de Higiene e Medicina Tropical, série II, vol 13, pp.387, [Article in Portuguese].

14. Ribeiro H, Ramos HC (1973) Research on the mosquitoes of Angola. The genus AedesMeigen, 1818 (Diptera:Culicidae). Check-list with new records, keys to females and larvae, distribution and taxonomic and bioecological notes. Anais do Instituto de Higiene e Medicina Tropical Vol I, nº $1-4$ (Separata): 107-137. 
15. Kokernot RH, Casaca VM, Weinbren MP, McIntosh BM (1965) Survey for antibodies against arthropod-borne viruses in the sera of indigenous residents of Angola. Trans R Soc Trop Med Hyg 59: 563-570.

16. Bakker RC, Veenstra J, Dingemans-Dumas AM, Wetsteyn JCFM, Kager PA (1996) Imported Dengue in The Netherlands. J Travel Med 3: 204-208.

17. Vasconcelos PF, Travassos da Rosa ES, Travassos da Rosa JF, de Freitas RB, Dégallier N, Rodrigues SG, Travassos da Rosa AP (1993) Outbreak of classical fever of dengue caused by serotype 2 in Araguaiana, Tocantins, Brazil. Rev Inst Med Trop São Paulo 35: 141-148, [Article in Portuguese].

18. Schwartz E, Meltzer E, Mendelson M, Tooke A, Steiner F, Gautret P, Friedrich-Jaenicke B, Libman M, Bin H, WilderSmith A, Gubler DJ, Freedman DO, Parola P (2013) Detection on four continents of dengue fever cases related to an ongoing outbreak in Luanda, Angola, March to May 2013. Euro Surveill 18: 20488.
19. Parreira R, Centeno-Lima S, Lopes A, Portugal-Calisto D, Constantino A, Nina J (2014) Dengue virus serotype 4 and chikungunya virus coinfection in a traveler returning from Luanda, Angola, January 2014. Euro Surveill19: pii 20730.

20. European Centre for Disease Prevention and Control (2014) Mosquito Maps. Available at: http://www.ecdc.europa.eu/en/healthtopics/vectors/vectormaps/Pages/VBORNET_maps.aspx. Accessed 10/August/2014.

\section{Corresponding author}

Ricardo Parreira

Unidade de Microbiologia Médica (Grupo de Virologia) and Unidade de Parasitologia e Microbiologia Médicas (UPMM), Instituto de Higiene e Medicina Tropical (IHMT), Universidade Nova de Lisboa (UNL), Rua da Junqueira 100, 1349-008 Lisboa, Portugal

Phone: +3 51213652600

Fax: + 3510213632105

Email: ricardo@ihmt.unl.pt

Conflict of interests: No conflict of interests is declared. 